\title{
Monte Carlo simulations of the randomly forced Burgers equation
}

\author{
P. DüBen ${ }^{1}$, D. Homeier ${ }^{1}$ (a), K. Jansen ${ }^{2}$, D. Mesterhazy ${ }^{3}$, G. MÜnster ${ }^{1}$ and C. Urbach ${ }^{3}$ \\ 1 Westfälische Wilhelms-Universität Münster \\ DESY, Zeuthen \\ Humboldt-Universität zu Berlin
}

PACS 02.70.Uu - Applications of Monte Carlo methods

PACS $05.20 . \mathrm{Jj}$ - Statistical mechanics of classical fluids

PACS $47.40 . \mathrm{Nm}$ - Shock wave interactions and shock effects

\begin{abstract}
The behaviour of the one-dimensional random-forced Burgers equation is investigated in the path integral formalism, using a discrete space-time lattice. We show that by means of Monte Carlo methods one may evaluate observables, such as structure functions, as ensemble averages over different field realizations. The regularization of shock solutions to the zero-viscosity limit (Hopf-eq.) eventually leads to constraints on lattice parameters, required for the stability of the simulations. Insight into the formation of localized structures (shocks) and their dynamics is obtained.
\end{abstract}

I. Introduction. - The random-force-driven Burgers equation

$$
\partial_{t} u+u \partial_{x} u-\nu \partial_{x}^{2} u=f
$$

may be interpreted as a model for compressible hydrodynamic turbulence, describing acoustic perturbations in the reference frame moving with the velocity of sound $[1,2]$. Here $u$ is the velocity, $f$ the random forcing, and $\nu$ is the kinematic viscosity. One generally assumes the force to be white-in-time, displaying Gaussian statistics

$$
\left\langle f(x, t) f\left(x^{\prime}, t^{\prime}\right)\right\rangle=\chi\left(x-x^{\prime}\right) \delta\left(t-t^{\prime}\right)
$$

so that the properties of the external pumping are completely characterized by the covariance $\chi$. The turbulent state is maintained by a large-scale external force with correlation length $L$. Finite $\nu>0$ and energy dissipation $\epsilon=\chi(0)$ provide a dissipation scale $\eta=\left(\nu^{3} / \epsilon\right)^{1 / 4}>0$. The dissipation is related to the Reynolds number Re by the definition $\epsilon \equiv \operatorname{Re}^{3} \nu^{3} / L^{4}$. Thus the characteristic velocity is $u_{L}=(\epsilon L)^{1 / 3}$. In the limit of large Reynolds number $\operatorname{Re}=(L / \eta)^{4 / 3} \gg 1$ these scales separate, so one expects a turbulent cascade. Evaluating moments of alilean invariant velocity differences $w(r) \equiv u(r)-u(0)$, structure functions

$$
S_{p}(r) \equiv\left\langle[w(r)]^{p}\right\rangle,
$$

$>0$, where $r$ is the displacement, one therefore expects a aling behavior in the inertial interval $L \gg r \gg \eta$. From dimensional considerations we find $S_{p}(r) \propto(\epsilon r)^{\zeta_{p}}$, and scale-invariance implies $\zeta_{p}=p / 3$. However, the formation of shocks in Burgers turbulence leads to a strong intermittency and a bifractal scaling of the form $\zeta_{p}=\min (p, 1)$, see e.g. [3].

We would like to emphasize that the average $\langle\cdots\rangle$ in Eq. (3) and in the following is usually considered to be an average over all times at a given place, or over all places at a given time; linked by the Taylor-hypothesis in statistically homogenous turbulence. In this article these averages are represented as ensemble averages, as discussed below.

Numerical investigations of hydrodynamic systems are commonly performed by means of direct simulations or variants thereof which amounts to integrating the equations of motion. In this article we follow a completely different approach, namely to evaluate the corresponding path integrals by Monte Carlo simulations. The clear physical picture of Burgers equation, and the fact that the intermittent structures are well-known - they correspond to shocks with a large negative velocity gradient - makes the Burgers equation an attractive setting to study Monte Carlo methods in turbulence theory. Further, a number of technical advantages leads to the unique possibility of investigating intermittent structures in turbulent flow. In particular, Burgers equation is local while incompressibility acts as a nonlocal interaction in Navier-Stokes turbulence which eventually complicates issues due the presence 
of the functional determinant arising in the path integral formulation of Eq. (1) (see Sec. II). Also the dissipation scale $\eta$ provides a UV-regularization of shock structures. Finally, a huge variety of analytical methods has been applied to Burgers equation, giving results that can directly be compared to numerical calculations. However, Burgers equation is certainly also interesting on its own (e.g. in cosmology), serving as model for systems with an interplay of random processes and coherent structures; for an overview see [3].

It is the aim of this paper to demonstrate the feasibility of Monte Carlo simulations in the path integral formalism. In particular we will show that respecting certain constraints on the parameters of the model, stable simulations can be performed. We will demonstrate with an example that this way structure functions can be computed precisely.

This paper proceeds as follows. In Sec. II we shortly introduce the path integral formulation for Burgers equation. Then in Sec. III the numerical scheme is discussed in detail and in Sec. IV the numerical results are given.

II. Path Integral for Burgers equation. - The path integral for the randomly forced Burgers equation is introduced via the Martin-Siggia-Rose formalism [4] where one has

$$
Z=\int D u D p \exp (-S[u, p])
$$

with the conjugate momenta $p$, and the action is $S[u, p]=\int d t \mathcal{L}[u, p]$. The Lagrangian is given by

$$
\mathcal{L}=-i \int d x p\left(\partial_{t} u+u \partial_{x} u-\nu \partial_{x}^{2} u\right)+\frac{1}{2} \int d x p(\chi * p),
$$

where $*$ denotes the convolution. Performing the Gaussian integration over the conjugate field, one arrives at

$$
\mathcal{L}=\frac{1}{2} \int d x N[u]\left(\chi^{-1} * N[u]\right)
$$

where $N[u] \equiv \partial_{t} u+u \partial_{x} u-\nu \partial_{x}^{2} u$. The functional determinant, arising in principle in the derivation of (4), (5) does not contribute to the forced Burgers equation due to the local interaction (see e.g. [5]). Thus the integration measure is properly defined, and the integration is over all fields $u$ (and $p$ ) in one dimension. In contrast, in the case of the Navier-Stokes equation, where the effect of pressure is not negligible, the corresponding non-local interaction leads to a non-vanishing functional determinant that has to be treated using the Fadeev-Popov procedure [6].

As (4), (5) are Galilean invariant, the computation of non-invariant quantities such as n-point correlation functions would require a gauge fixing [7]. However, since we are interested in evaluating structure functions, i.e. manifestly Galilean-invariant quantities, this procedure does not have to be performed.
Then the expectation values of Galilean invariant observables $O[u(x)]$ are calculated as ensemble-averages

$$
\langle O[u]\rangle=\frac{\int D u O[u] \exp (-S[u])}{\int D u \exp (-S[u])} .
$$

Extending the time integration in the action to infinity renders the averages stationary (see e.g. [8]).

Using the path integral (4),(5) it has been shown that intermittent statistics of Burgers equation can be understood in terms of instanton solutions [9-11]. These solutions break Galilean invariance which has been shown to account for intermittency [12].

III. Monte Carlo Simulations. - Using the path integral formalism we calculate ensemble averages like (7) numerically, where the Lagrangian is given by (6). Defining the theory on a finite size lattice, the integration is performed over grid variables $u_{i}(n)$, i.e. the field $u$ defined at lattice sites $(i, n)$ where $i$ denotes the discretized space, and $n$ discretized time points. Then we obtain

$$
\langle O\rangle=\int \prod_{i, n} d u_{i}(n) O\left[\left\{u_{i}(n)\right\}\right] \exp \left(-S\left[\left\{u_{i}(n)\right\}\right]\right)
$$

for the observable computed in the discretized theory. Here the discretized action $S\left[\left\{u_{i}(n)\right\}\right]$ may be so chosen that the single-site action $S\left(u_{i}(n)\right)$ is quadratic:

$$
S\left(u_{i}(n)\right)=A_{i}(n)\left[u_{i}(n)-C_{i}(n)\right]^{2}+B_{i}(n),
$$

where $A_{i}(n)>0$. The terms $A_{i}(n), C_{i}(n)$ and $B_{i}(n)$ will in general depend on the field at different nodes on the lattice. The action is locally minimized by choosing $u_{i}(n) \simeq C_{i}(n)$ thus giving the main contribution to the integral in (8). This way one may evaluate the whole integral, by successively integrating over different lattice sites. Successive application of such sweeps, will then generate the Markov chain $\left\{u_{i}^{(k)}(n)\right\}$ of configurations in the $k$-th step of the process. The quadratic form of the action in (9) allows to apply a heat-bath Monte Carlo algorithm to update single field variables on the lattice. However, it should be emphasized that writing the single-site action as (9) imposes a restriction on the possible form of the nonlinear term $u \partial_{x} u$. In its discretized version, it has to be at most linear in $u_{i}(n)$. Thus we are led to define the nonlinear term as $u \partial_{x} u \rightarrow u_{i}(n) u_{i}^{\prime}(n)$, where the derivative is written symmetrically with the simplest choice being $u_{i}^{\prime}(n)=\left(u_{i+1}(n)-u_{i-1}(n)\right) / 2 \Delta x$. The time derivative and the viscous term are discretized in the usual way.

The above action was put onto a rectangular lattice with $N_{\mathrm{X}}$ sites in space- and $N_{\mathrm{t}}$ sites in time-direction. Lattice spacings are denoted correspondingly by $\Delta x$ and $\Delta t$. Monte Carlo simulations were performed for different lattice sizes, ranging from e.g. $\left(N_{\mathrm{X}}=16\right) \times\left(N_{\mathrm{t}}=4\right)$, to larger lattices of up to approximately $\left(N_{\mathrm{X}}=362\right) \times\left(N_{\mathrm{t}}=4096\right)$ at fixed viscosity respectively. The Reynolds number ranged from $\operatorname{Re}=2$ to $\operatorname{Re}=16$. 
Lattice parameters and Continuum Limit. In our simulations we chose the forcing characteristics as

$$
\chi\left(x-x^{\prime}\right)=\chi(0) \exp \left(-\left|x-x^{\prime}\right| / L\right),
$$

in agreement with the considerations in [10]. In Fourierspace this represents a noise $\tilde{\chi}(k)$ with an IR-cutoff at wave-number $k \simeq L^{-1}$ and a power-law behavior for $k \gg L^{-1}$, of the form $\chi(0) L^{1+\beta} k^{\beta}$, where $\beta=-2$. That is a random force acting predominantly at large scales $\gtrsim L$, leading to shock-formation at random positions in our system [3].

To identify the lattice parameters with the corresponding constants of the continuum theory, first notice that the viscosity has to be defined as

$$
\nu \equiv \tilde{\nu}(\Delta x)^{2} / \Delta t
$$

where the arbitrary constant $\tilde{\nu}$ has been introduced, relating units for space- and time-measurements on the lattice. This can be shown by performing the continuum limit in the case of the symmetric random walk, leading to the diffusion equation. The so-defined $\nu$ gives us the Reynoldsnumber Re according to the earlier given relations, after $\chi(0)$ and $L$ have been specified. The continuum limit is then performed by $\Delta x, \Delta t \rightarrow 0$ while keeping $L, \nu$ and $\operatorname{Re}$ constant.

In both space- and time-direction periodic boundary conditions were specified. Then one may expect that in the continuum limit the correct physical behavior of the fields is assumed and correlation effects induced by the boundary conditions are negligible.

Stability considerations lead to further constraints for the lattice size, as will be explained in the following subsection.

Stability and Regularization. As one would expect, the stability of the simulations over a large number of Monte Carlo steps is a big issue. Indeed, if certain restrictions on the lattice parameters are not taken care of, the simulation terminates sooner or later due to instabilities.

The occurence of instabilities in our Monte Carlo simulations is related to the existence of shock-like solutions of Eq. (1). To obtain stable simulations, the shock structures have to be resolved on the lattice, otherwise the action near the shock positions is represented badly and the simulation tends to develop instabilities, accumulating the overall energy of the configurations near the shocks.

In the limit of small viscosities it would be possible to regularize the shocks by adding higher derivative terms or nonlinear terms to the differential equation. Similar to the case of the Korteweg-de Vries equation, where the term proportional to $\partial_{x}^{3} u$ serves to regularize shock waves $[13,14]$, the Burgers equation can be regularized by adding e.g. terms proportional to $\partial_{t} \partial_{x}^{2} u$ and $u \partial_{x}^{3} u$ to it [15]. This would smear out the shocks and enable their proper treatment in the simulation. As indicated by the shock solution of the unforced Burgers equation

$$
u=-A \tanh \frac{A}{2 \nu} x,
$$

the viscosity term itself provides a valid regularization of shocks. Therefore we decided to smooth out shocks by chosing the viscosity appropriately. This amounts to resolve the dissipation scale $\eta$ on the lattice. In terms of the Reynolds number this translates into the constraint

$$
\operatorname{Re} \lesssim L N_{\mathrm{X}}
$$

At given Reynolds number Re $\gg 1$ and $L<1$ this imposes a strong restriction on the possible lattice sizes.

Respecting the constraints on the parameters we are in the position to perform stable simulations. In particular, we can resolve a shock on our lattices as Fig. (1) demonstrates, which shows a time-slice of a typical configuration. We also can observe time dynamics in our simulations as Fig. (2) shows, and finally, we are able to compute the structure functions an example of which is given in Fig. (3).

IV. Summary and Outlook. - We have shown how to perform stable Monte Carlo simulations of stochastic partial differential equations like the Burgers equation in the path integral formulation, and in this way demonstrated that it is possible to calculate observables like the structure functions using an ensemble-average rather than a time- or space-average. The lattice versions of the theories can directly be identified with their continuum counterparts, and as long as certain constraints on the lattice size are respected no instabilities occur. Direct insight into the structures leading to intermittency and, thus, multiscaling, can be obtained. Especially, we want to point out that the existence of a dissipation length scale can be observed.

In particular in our simulations of the randomly forced Burgers equation we observe the following:

- Thermalization and autocorrelation times are very long, up to the order of $10^{5}$ Monte Carlo steps.

- In the stable runs after thermalization, the typical shock solutions of Burgers equation and their dynamics can be observed, see Fig. (1) and Fig. (2).

- In the unstable runs before occurence of instabilities, configurations show typical shock solutions leading eventually to an energy accumulation near shock positions.

- The distinction between stable and unstable simulations can directly be related to the existence of a dissipation length scale which is either bigger (stable) or smaller (unstable simulations) than the lattice spacing.

First results on the extracted structure functions, e.g. see Fig. (3) are promising. However, the understanding of systematic errors such as finite size effects or fitting ranges for extracting the scaling exponents from the measured 


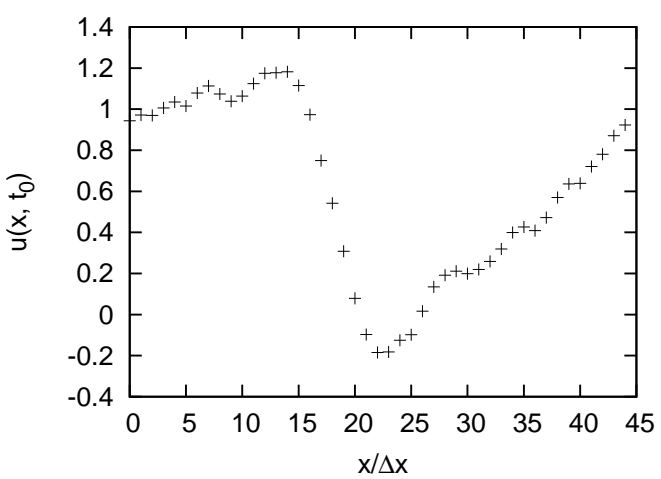

Fig. 1: Slize of a typical configuration $u\left(x, t_{0}\right)$ at constant time $t_{0}$, depending on the (spatial) lattice site $x / \Delta x$; taken from a $N_{\mathrm{X}}=256, N_{\mathrm{t}}=45$ lattice where $\operatorname{Re}=4, \nu=1 / 32$. The typical kink solution associated with the Hopf-eq. can be clearly seen, including a finite shock width due to regularizing viscosity.

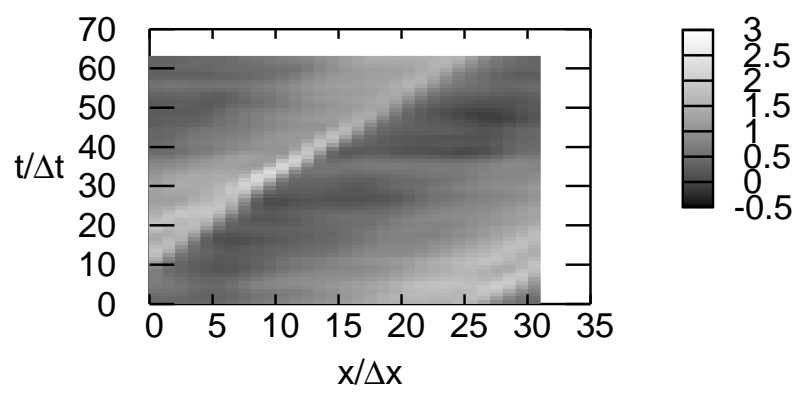

Fig. 2: 3D-Plot of a configuration $u(x, t)$; taken from a $N_{\mathrm{X}}=$ $32, N_{\mathrm{t}}=64$ lattice. The propagation of the shock with constant velocity is clearly visible.

structure functions is still in progress. Nevertheless, we conclude that the path integral Monte Carlo approach to the Burgers equation outlined in this article is a feasible and stable tool to obtain interesting numerical results.

This work is supported by the Deutsche Forschungsgemeinschaft under project no. Mu 757/14.

\section{REFERENCES}

[1] Burgers J., The nonlinear Diffusion Equation: Asymptotic solutions and statistical problems (Reidel, Dordrecht) 1974.

[2] Falkovich G., Introduction to turbulence theory presented at Non-equilibrium Statistical Mechanics and Turbulence, edited by NAZARENKO S. and ZABORONSKI O. V., London Mathematical Society Lecture Note Series 355 (Cambridge Univ. Press.) 2008.

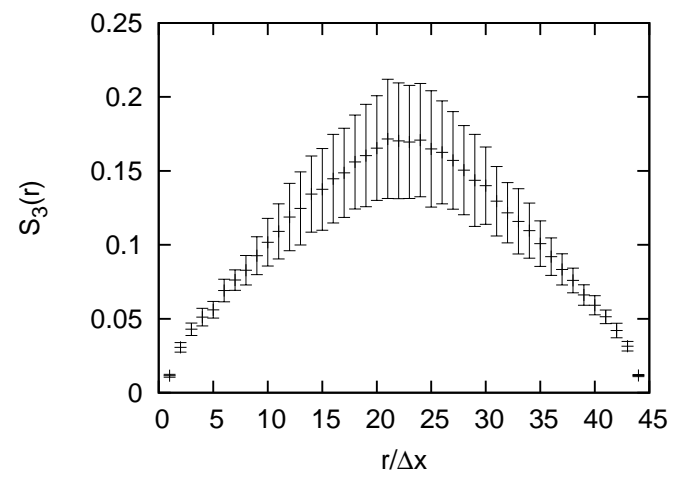

Fig. 3: Third order structure function $S_{3}(r)$ as a function of the separation $r / \Delta x$, evaluated from a simulation with $5 * 10^{6}$ configurations on a $N_{\mathrm{X}}=256, N_{\mathrm{t}}=45$ lattice with $\mathrm{Re}=$ $16, \nu=1 / 512$.

[3] BEC J. and KHANIN K., Burgers turbulence arXiv:0704.1611v1 [nlin.CD] (2007).

[4] Martin P., Siggia E. and Rose H., Phys. Rev. A , 8 (1973) 423.

[5] Hochberg D., Molina-París C., PÉrez-Mercader J. and Visser M., Phys. Rev. E , 60 (1999) 6343.

[6] HomeIER D., Renormierungsgruppenflussgleichungen und hydrodynamische Turbulenz (Ph.D. thesis) 2006.

[7] Berera A. and Hochberg D., Phys. Rev. Lett. , 99 (2007) 254501.

[8] Gozzi E., Phys. Rev. D, 28 (1983) 1922.

[9] Falkovich G., Kolokolov I., LebedeV V. and Migdal A., Phys. Rev. E , 54 (1996) 4896.

[10] Balkovsky E., Falkovich G., Kolokolov I. and Lebedev V., Phys. Rev. Lett. , 78 (1997) 1452.

[11] Victor G. and Alexander M., Phys. Rev. E , 54 (1996) 4908.

[12] Polyakov A., Phys. Rev. E , 52 (1995) 6183.

[13] Lax P. and Levermore C., Comm. Pure Appl. Math. , 36 (1983) 253,571,809.

[14] Venakides S., Comm. Pure Appl. Math. , 43 (1990) 335.

[15] Bhat H. and Fetecau R., J. Nonlinear Sci. , 16 (2006) 615 . 